\title{
El negocio de buscar en internet. Análisis del mercado de los buscadores en 2003
}

\author{
Por Jordi Grau Moracho y Javier Guallar Delgado
}

\begin{abstract}
LOS BUSCADORES DE INTERNET se han convertido en pocos años en un punto de referencia imprescindible para la obtención de todo tipo de información. Representan las verdaderas páginas amarillas de la Red. Su grado de presencia en los hábitos telemáticos de la población es tan grande que resulta difícil imaginar una sesión cualquiera en internet sin la utilización de un buscador.
\end{abstract}

Paralelamente a su uso creciente, en los últimos años se observa una progresiva concentración de las búsquedas en unas pocas marcas. Si bien existe un importante número de sitios web donde buscar cada vez son menos las alternativas que se reparten la mayor parte de los resultados, es decir, cada vez son menos los indexadores reales de páginas de la Red. Entre las circunstancias causantes de esta situación se pueden citar al menos tres: la crisis de la denominada "burbuja tecnológica", que dio al traste con muchas compañías del sector; la tendencia empresarial hacia la concentración mediante fusiones o adquisiciones; y el sorprendente y avasallador éxito de algunas empresas (en particular, Google).

\section{Evolución de los buscadores y sus estrategias de negocio}

Los buscadores han tenido siempre un elevado protagonismo en la breve historia de la world wide web. A mediados de los $90 \mathrm{Al}$ tavista, como motor de búsqueda fulltext automatizado, y Yahoo, como directorio organizado manualmente, representaron los dos modelos principales de acceso a la información de internet, que muchos

imitaron. En ambos casos, la financiación del servicio, gratuito para los usuarios, se hacía mediante la publicidad insertada en las páginas web.

Durante el denominado boom tecnológico, ambos sistemas evolucionaron hacia un nuevo concepto denominado portal, basado en la oferta de múltiples servicios a sus visitantes. En los portales, las búsquedas de información forman parte de la oferta diversificada del sitio junto a chats, foros, noticias o direcciones de correo electrónico de acceso gratuito. Los modelos de negocio para rentabilizar la creciente inversión en infraestructuras y servicios, siempre gratuitos para el visitante, siguen basándose en la venta de publicidad en diferentes formatos.

\section{«La globalización en el mercado se debe a la crisis tecnológica, que acabó con mu- chas compañías del sector; la tendencia empresarial hacia la concentración me- diante fusiones o ad- quisiciones; y el ava- sallador éxito de al- gunas empresas co-
mo Google»}

El auge de Google, desde el año 2000 , con una vuelta al modelo original de buscador "puro" desplazó de su lugar de privilegio a algunos veteranos, como Altavista, Infoseek o Excite hasta convertirlo en el protagonista indiscutible del sector. En la actualidad, coexisten

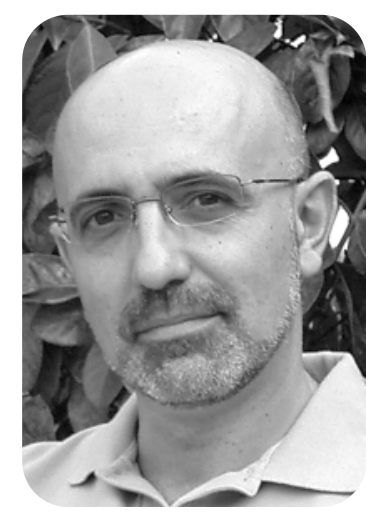

Jordi Grau y Javier Guallar

en el mercado dos tipologías de sitios web de búsquedas: los buscadores "puros" (Google, Altavista, Ask Jeeves, Alltheweb) y los portales (Yahoo, MSN, AOL, Lycos).

En ese contexto, Overture ha introducido una nueva estrategia comercial, a la que Google se ha sumado con gran rapidez de reflejos: la venta de posicionamiento en los resultados de las búsquedas. El sistema conoce en la actualidad varias denominaciones. Las más habituales en la bibliografía en inglés son "pay per click" (PPC), "paid listings" y "sponsored links", mientras que en español se suele hablar de "resultados previo pago" o de "enlaces patrocinados". La estrategia en la página de resultados de los buscadores va postergando los mensajes publicitarios en forma de banners, pop-ups y propuestas más agresivas para dejar paso a un procedimiento más sutil: estar en los primeros lugares de la lista bajo un determinado término de búsqueda. La clave, aunque bajo distintas presentaciones, es que la publicidad pase lo más desapercibida posible.

Este último año, con la paulatina concentración empresarial del sector, la evolución de ese modelo comercial basado en la venta de relevancia se ha hecho realidad y ha 


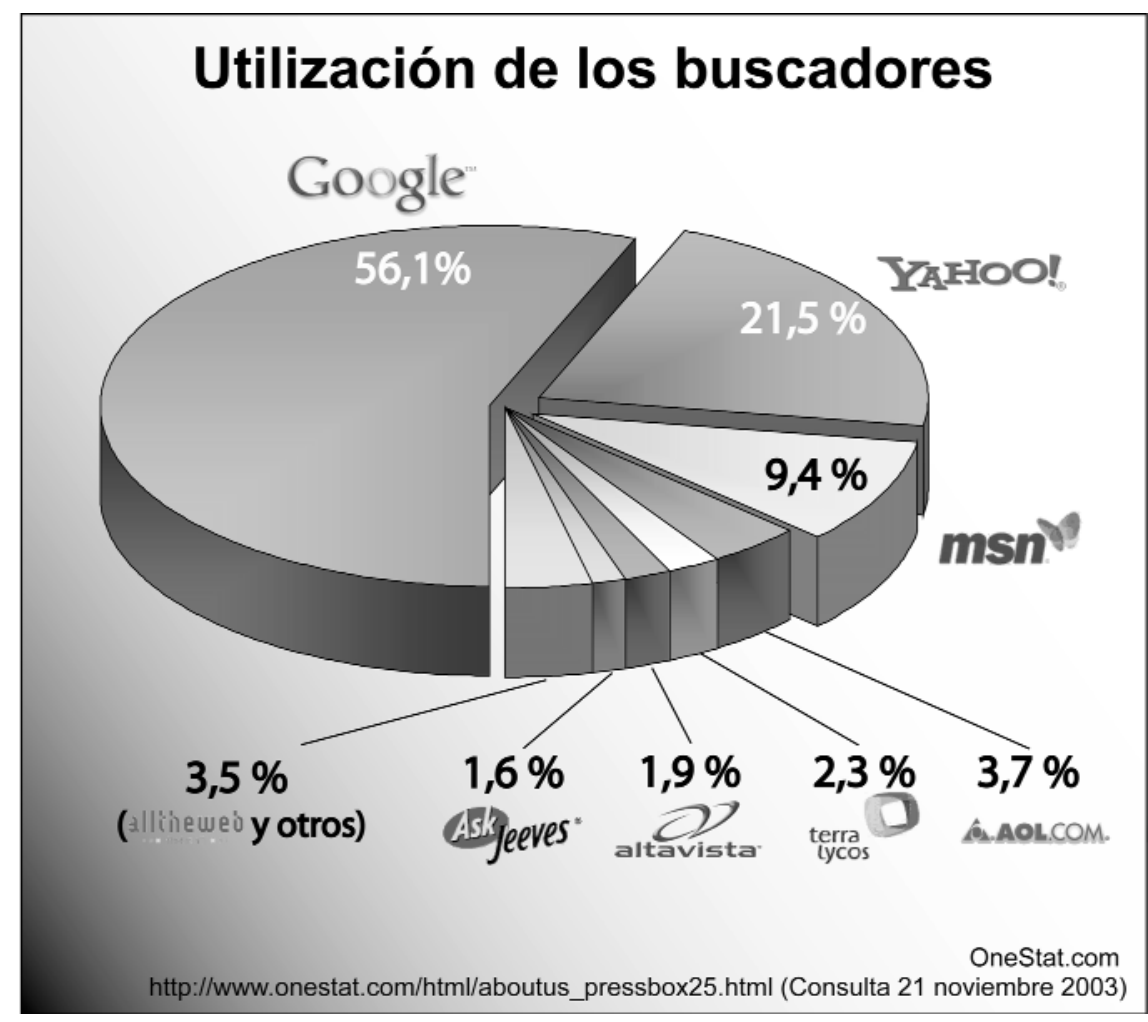

comenzado a dar sus frutos. Los enlaces patrocinados, a unos pocos céntimos el impacto, han resultado ser un suculento negocio.

\section{Un pastel en pocas manos}

Una observación atenta del mercado en 2003 permite señalar algunas tendencias: la consolidación absoluta de Google como referencia del sector; el aumento de la concentración empresarial debido en especial a las importantes adquisiciones realizadas por Yahoo que han sacudido continuamente el mercado; y los avisos de Microsoft, desde los rumores de una posible adquisición de Google al anuncio del desarrollo de un nuevo motor de búsqueda.

Para conocer el grado de utilización de los buscadores se pueden consultar los datos que presentan periódicamente algunas empresas que realizan mediciones del tráfico de internet, como One Stat o Nielsen Net Ratings

http://www.onestat.com

http://www.nielsen-netratings.com to, $3,5 \%$.
Los porcentajes muestran el dominio acaparador de Google, que no solamente se ha consolidado de manera destacada en 2003 como el buscador número uno, sino que día a día ha aumentado su presencia en la Red. Más de la mitad de las búsquedas en internet se hacen con Google: nada menos que un $56,1 \%$, habiendo subido casi un punto porcentual en poco tiempo, ya que en la medición de One Stat efectuada seis meses antes tenía un 55,2. Más aún, esta cifra todavía es mayor si se toman en consideración las búsquedas realizadas en otros sitios web con su motor, como es el caso de $A O L$ o de su principal rival, Yahoo.

El hecho es que el éxito del buscador fundado en 1996 por Larry Page y Sergei Brin es tan grande que su nombre se ha convertido en sinónimo de buscar en la Red, como pone de manifiesto la popularización en el ámbito anglosajón del término googling. Durante 2003 , no ha parado de crecer en usuarios mientras miraba de reojo a sus rivales y permanecía fiel a sus señas de identidad: la investigación en tecnología. Sus creadores saben que la clave de su hegemonía se llama Page Rank, la tecnología de presentación de los resultados de las búsquedas basada

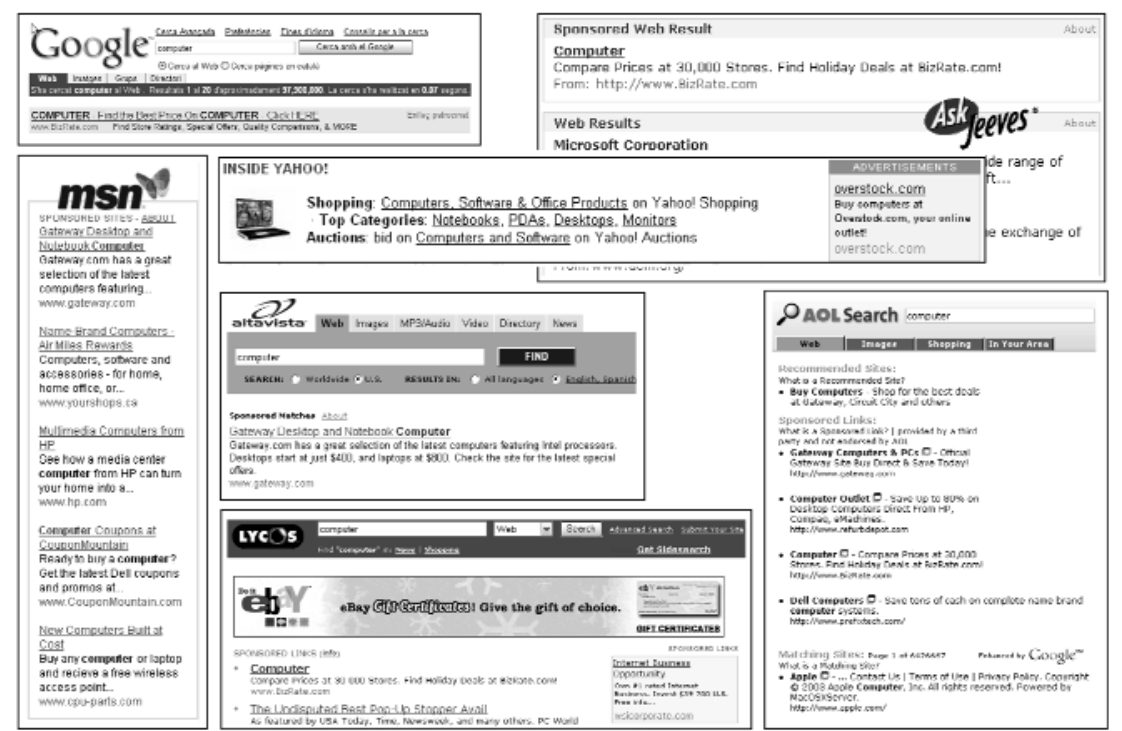

Mosaico de buscadores 


\section{Cronología del mercado de búsquedas en internet en 2003}

Diciembre 2002. Google pone en marcha Froogle, un buscador de productos que se venden a través de internet, y Google News, que selecciona de manera automática noticias de unos 4000 medios de comunicación.

Diciembre 2002. Yahoo anuncia la adquisición del motor de búsqueda Inktomi por 365 millones de dólares.

Febrero 2003. Google adquiere la compañía informática Pyra, creadora del servicio de weblogs Blogger.

Febrero 2003. Overture anuncia la compra de la compañía propietaria del buscador Altavista por 140 millones de dólares. Pocos días después, lleva a cabo una segunda adquisición de trascendencia al comprar el área de internet de la compañía Fast -que incluye el buscador Alltheweb -, por 100 millones de dólares.

Marzo 2003. El buscador Altheweb, adquirido por Overture, renueva su diseño, cambiando su aspecto y añadiendo mejoras en las búsquedas.

Marzo 2003. El portal AOL renueva su diseño e incorpora en sus búsquedas los resultados proporcionados por Google.

Marzo 2003. M icrosoft incorpora nuevas aplicaciones y mejoras en su buscador M SN Search.

Abril 2003. Yahoo presenta un renovado motor de búsqueda en la línea de su principal competidor Google

Abril $\mathbf{2 0 0 3}$. Ask Jeeves presenta también la renovación de su buscador.

Abril 2003. Google anuncia la compra de la empresa Applied Semantics.

Junio 2003. Google presenta la nueva versión de su barra de navegación, la Google Toolbar 2.0, que bloquea la aparición de pop-ups.

Julio 2003. Yahoo realiza la gran adquisición empresarial del año: compra la compañía Overture por 1.630 millones de dólares en efectivo y acciones.

Octubre 2003. Microsoft anuncia que su buscador MSN dejará de utilizar los resultados de LookSmart a partir de enero de 2004.

Octubre 2003. AOL y Google amplían su acuerdo de provisión de servicios de búsquedas y enlaces patrocinados.

Noviembre 2003. M icrosoft desmiente las noticias aparecidas en la prensa norteamericana sobre su interés en una adquisición de Google, presenta el buscador de noticias MSN N ewsbot y anuncia que está probando un nuevo motor de búsqueda de tecnología propia denominado MSN bot.

Noviembre 2003. Google anuncia una próxima salida a bolsa para marzo de 2004, que incluiría una subasta de acciones por internet.

Diciembre 2003. A parición de la versión beta de Google Print, que permite buscar en el contenido de unos 60.000 libros.

Diciembre 2003. M icrosoft anuncia el próximo lanzamiento de su propia barra de herramientas a imitación de la de Google, la M SN Toolbar. en un algoritmo matemático que ellos idearon y que ha resultado hasta ahora la mejor o la más efectiva. Pero el reto, claro está, es seguir siéndolo.

En este sentido se pueden entender las adquisiciones realizadas de algunas empresas pequeñas, a diferencia de Yahoo, pero significativas en cuanto a innovación tecnológica, como es el caso de Pyra, creadora del servicio de bitácoras Blogger, con el objetivo de gestionar mejor la operatividad en el sector emergente de las bitácoras o weblogs, o de Applied Semantics, especializada en "tecnologías de proceso semántico de textos y publicidad online", en palabras de Brin.

"Yahoo ha sido constantemente noticia a lo largo del último año, con una agresiva política de adquisiciones en el sector de las búsquedas desde que en diciembre de 2002 anunciara la compra de Inktomi»

Por otro lado, su sitio web, sin abandonar la simplicidad marca de la casa, continúa mejorando su diversificación de servicios de búsquedas, con nuevas aportaciones como el buscador de noticias Google News. La otra gran novedad ya señalada es el éxito de su posicionamiento en el sector de los enlaces patrocinados en competencia directa con Overture, hasta el punto de que éstos constituyen su principal fuente de ingresos en estos momentos. Para el futuro, ya ha anunciado algunos proyectos, como un nuevo buscador de imágenes, otro de archivos multimedia y el más inmediato —disponible desde diciembre de 2003 en versión beta- denominado Google Print, un novedoso servicio para 


\section{SISTRMA SABINI}

\section{Soluciones integrales para la Automatización de Bibliotecas y Centros de Documentación}

Desarrollado integramente en castellano con más de 15 años de experiencia en España y

Latinoamérica, atiende las necesidades de todo tipo y tamaño de bibliotecas y centros de información

\section{MÓDULOS:}

- Adquisiciones

Trámite de pedidos
Gestion de gastos y proveedores

- Catalogación

Catalogación de todos los materiales bibliográficos Integración de información bibliográfica

- Terminologia

Ficheros de autoridades Tesauro multilingúe

- Consultas

Lenguaje de Comandos

Acoeso público en linea (CAPEL)

Acceso a través de WWW

Protocolo Z39.50

- Circulación

Gestion de préstamos

Fichero de usuarios

- Estadisticas

De proceso y circulación

\section{Otros servicios:}

Instalación y soporte técnico del sistema SABINI

Asesoría en Automatización de Bibliotecas y Centros de Documentación

Procesamiento de material bibliográfico

Instalación de catálogos en Internet de Bibliotecas
C. Amor de Dios, $n^{*} 1$

Telt: +34 91 428355

Fax +34914292610

28014 MADRD

o-mait sabiriessabinicom

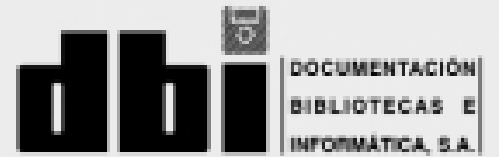

Av. Diez Cansece 236 of. 602 Lima 18 Teletax (511) 446-0815 e-mail: sabini Glerra compe 
buscar en el contenido de unos 60.000 libros.

Desde esta situación hegemónica, la empresa ha confirmado recientemente una noticia largamente esperada: su próxima salida a bolsa. El anuncio ha puesto fin, al menos momentáneamente, a toda suerte de especulaciones existentes los últimos meses sobre el interés del gigante Microsoft en su adquisición. Sin duda, la entrada del buscador número uno en el mercado bursátil será una de las noticias relevantes de 2004 que puede condicionar en gran medida la posterior evolución de todo el sector.

El predominio de Google debe preocupar enormemente a la competencia, que no puede dormirse si quiere como mínimo mantener su actual nivel de presencia. Yahoo y Microsoft se han diferenciado con claridad del resto, y presentan también unos niveles considerables de penetración en el mercado.

"Google no solamente se ha consolidado de manera destacada como el buscador número uno de 2003 , sino que día a día sigue aumentado su presencia en la Red»

Por un lado, Yahoo, una de las firmas más veteranas y de mayor prestigio para los internautas, ha sido constantemente noticia a lo largo del último año, con una agresiva política de posicionamiento en el sector de las búsquedas desde que en diciembre de 2002 anunciara la adquisición de Inktomi. Identificado originalmente con su popular directorio, hoy es un gran portal con una estrategia comercial $\mathrm{y}$ de servicios muy diversificada, en el que su función de buscador cobra progresiva relevancia. En este año ha potenciado y diferenciado más claramente su oferta de búsqueda con su Yahoo Search si- guiendo el modelo de simplicidad de Google y ha adquirido nada menos que Overture, la empresa pionera en el negocio de los enlaces patrocinados, que además acababa de adquirir a su vez en poco tiempo dos de los mejores motores de búsqueda de internet, Altavista y Fast (Alltheweb).

Los datos son evidentes y hablan por sí solos. Firmas de gran peso en el sector como Inktomi, Overture, Altavista y Fast (Alltheweb), que en diciembre de 2002 eran todas ellas empresas independientes están un año después en sus manos. Aunque en la actualidad siga pagando a Google por la utilización de su motor de búsqueda, con todo este enorme potencial que ha acumulado en pocos meses, Yahoo aparece situada en un sólido segundo lugar, a la espera de integrar todas las diferentes tecnologías en su poder y poder superar así la dependencia respecto a Google.

Microsoft por su parte ocupa el tercer puesto del ranking de One Stat con su MSN Search, en una situación que se puede considerar discreta en relación con la hegemonía superior al $95 \%$ que mantiene en el sector del software de uso personal. Con todo, su nivel de presencia es superior a las prestaciones reales de su buscador, debido sin duda a esa posición dominante en el software doméstico, que ha sabido utilizar en su beneficio aunque sea recurriendo a algunas argucias, como el envío por defecto de los usuarios del Explorer a la página del buscador $M S N S e$ arch cada vez que el navegador detecta un error de página no encontrada.

Preocupada por el crecimiento de Google, la compañía ha dado algunas pistas de que prepara alguna acción sonada inminente. En los últimos meses se ha especulado insistentemente sobre la existencia de contactos encaminados a la adquisición de Google, si bien en es- tos momentos sus gestores, con el propio presidente Bill Gates a la cabeza, han manifestado su interés en el desarrollo de un nuevo motor de búsqueda propio de altas prestaciones.

«El modelo comercial basado en la venta de relevancia se ha hecho realidad. Los enlaces patrocinados, a unos pocos céntimos el impacto, han resultado ser un suculento negocio»

En resumen, estas tres empresas acaparan el $87 \%$ del tráfico mundial de búsquedas en sus propios sitios web, aunque el porcentaje puede ser superior si tenemos en cuenta los resultados indirectos a través del suministro de búsquedas a otros portales y las últimas adquisiciones. Estas cifras, de seguir en aumento, cuestionan la diversidad de oferta que caracterizaba la Red antes del ascenso imparable de Google.

En el ranking de One Stat aparecen cuatro nombres más. Los dos siguientes, AOL y Terra-Lycos, son grandes portales que ofrecen una amplia oferta de servicios a sus visitantes. En los dos casos, contratan la tecnología para la realización de las búsquedas a terceros (a $G o$ ogle y Fast respectivamente). El tercero es el histórico motor Altavista, ahora en manos de Overture y Yahoo.

Finalmente, Ask Jeeves es la única empresa independiente con tecnología propia de búsquedas, más allá de las tres grandes, y sólo el tiempo dirá si conseguirá consolidar su espacio en este difícil mercado. En este sentido, su presidente Steve Berkowitz ha reivindicado sus opciones - hay que subrayar que el motor Teoma de Ask Jeeves tiene una alta consideración entre los especialistas- al mani- 
festar que las búsquedas web están todavía en su infancia y que la tecnología resulta aún demasiado imperfecta, por lo que queda todavía mucho camino por recorrer.

\section{Interdependencia empresarial}

Para completar la visión del panorama descrito es interesante observar las relaciones que se establecen en el mercado de los buscadores entre los sitios web, los motores de búsqueda y los proveedores de enlaces patrocinados.

En la tabla se muestran en la columna izquierda de la tabla los webs más visitados como buscadores (a los siete primeros del ranking de One Stat se ha considerado oportuno añadir Alltheweb), relacionados respectivamente con sus empresas propietarias, con los motores que realizan las búsquedas en dichos sitios y con las compañías que comercializan los enlaces esponsorizados.

Solamente Google y Altavista se autoabastecen completamente y pueden ofrecer resultados propios a sus usuarios. En todos los demás casos, existe una interdependencia empresarial entre el sitio, el motor y el pay per click.

Así, los portales Yahoo, AOL y Lycos ofrecen servicios de búsquedas a sus usuarios pero compran a terceras empresas el derecho del uso de su tecnología y sus bases de datos (Google y Fast). Es decir, pagan para disponer de sistemas de búsqueda con el objetivo de atraer usuarios, lo que atrae a su vez a anunciantes a los que vender relevancia en sus páginas de resultados.

Por su parte, los motores de búsqueda tradicionales con tecnologías propietarias como Google y Fast, indexan los contenidos de internet $\mathrm{y}$ venden sus resultados a portales como Yahoo, AOL y $\mathrm{Ly}$ $\cos$, que pagan por cada resultado que se realiza a través su sitio. Por ejemplo, en al año 2001 Google pagó a Yahoo 1 millón de dólares por publicidad y branding, pero cobró 7,5 millones en concepto de fees por utilización del motor de búsqueda.

Por último, el negocio para las firmas que controlan el mercado del pay per click (Google y Overture) se basa en la comercialización de sus bases de datos de clientes en los webs de búsquedas.

\section{Cómo funcionan los enlaces patrocinados}

Cuando un usuario de un buscador lanza una consulta cualquiera recibe en cuestión de segundos una lista ordenada de resultados. Junto a los enlaces que obedecen a criterios objetivos de relevancia

\begin{tabular}{|c|c|c|c|}
\hline Sitio web & Empresa & Motor & $\begin{array}{c}\text { Enlaces } \\
\text { patrocinados }\end{array}$ \\
\hline
\end{tabular}

\begin{tabular}{|c|c|c|c|}
\hline $\begin{array}{c}\text { Alltheweb } \\
\text { http://www.alltheweb.com }\end{array}$ & $\begin{array}{c}\text { Overlure } \\
\text { (Yahoo) }\end{array}$ & Fast & $\begin{array}{c}\text { Overture (EUA), } \\
\text { Espotting (Europa) }\end{array}$ \\
\hline $\begin{array}{c}\text { Altavista } \\
\text { http://www.altavista.com }\end{array}$ & $\begin{array}{c}\text { Overture } \\
\text { (Yahoo) }\end{array}$ & Altavista & Overture \\
\hline $\begin{array}{c}\text { AOL Search } \\
\text { http://www.aol.com }\end{array}$ & America Online & Google & Google \\
\hline $\begin{array}{c}\text { Ask Jeeves } \\
\text { http://www.ask.com }\end{array}$ & Ask Jeeves & Teoma & Google \\
\hline $\begin{array}{c}\text { Google } \\
\text { http://www.Google.com }\end{array}$ & Google & Google & Google \\
\hline $\begin{array}{c}\text { MSN Search } \\
\text { http://www.msn.com }\end{array}$ & Microsoft & $\begin{array}{c}\text { MSN/ } \\
\text { LookSmart }\end{array}$ & Overture \\
\hline $\begin{array}{c}\text { Lycos } \\
\text { http://www.lycos.com }\end{array}$ & Terra Lycos & Fast & Overture \\
\hline $\begin{array}{c}\text { Yahoo } \\
\text { http://www.yahoo.com }\end{array}$ & Yahoo & Google & Overture \\
\hline
\end{tabular}

Relaciones entre los sitios web de búsquedas y sus proveedores de resultados (frecuencia absoluta, ubicación, densidad, inmediatez, popularidad o proximidad), dependiendo del web utilizado se muestran más o menos diferenciados y priorizados otros enlaces con la etiqueta "patrocinados" o simplemente "Overture".

Los buscadores perciben sus ingresos principalmente de este tipo de publicidad que representa para sus clientes (empresas, organizaciones, partidos políticos, instituciones, etc) poder aparecer en una mejor situación en la lista de resultados. Para las organizaciones que pagan por situarse en las primeras posiciones, ésta es una de las formas más efectivas de publicitar un web en internet.

Los anunciantes pujan una cantidad, que parte de unos pocos céntimos, por cada palabra clave por la que quieren ser encontrados. Dependiendo del precio que tenga ese término aparecerán más arriba o más abajo de la lista. Si muchos clientes pujan por la misma el precio aumentará y, si por el contrario, es poco demandada, con menos dinero aparecerá en los primeros lugares. Los diferentes anunciantes interesados en una palabra son ordenados rigurosamente según la cantidad que han pagado.

«Los anunciantes pu-

jan una cantidad, que parte de unos pocos céntimos, por cada palabra clave por la que quieren ser en-
contrados»

Todo está previsto, pues si dos clientes ofertan lo mismo se sigue un criterio de antigüedad o si uno de ellos ofrece un precio excesivamente superior al de su inmediato perseguidor puede ser informado por los administradores del buscador para que lo ajuste y reducir así la brecha de coste. Asimismo, si un competidor puja para aventajar a 
su rival, éste dispone de un mecanismo por el que puede aumentar su licitación hasta un determinado nivel máximo especificado. Tanto Google como Overture hacen un seguimiento de la competencia y reducen automáticamente el coste por clic real $(P P C)$ para que el anunciante pague el mínimo precio posible por mantener la posición de su anuncio en la página.

Cada buscador dispone de herramientas para que el cliente pueda gestionar sus licitaciones, los términos por los que quiere ser encontrado, la cantidad límite que quiere gastar, las fechas en las que realizará sus campañas, los países y los idiomas en los que se realizará. Otras funcionalidades ofrecen soporte para buscar palabras clave o frases con los sinónimos más populares sobre la base de miles de millones de búsquedas de los usuarios. Esto hace que el anunciante obtenga una mejor segmentación y un mayor número de impactos.

Las ingentes estadísticas que disponen las empresas de PPC o de paid listings sobre las búsquedas realizadas por los usuarios permiten realizar estimaciones sobre las campañas con una gran facilidad. Al seleccionar las palabras clave, se presentan automáticamente al cliente cálculos aproximados del número diario de peticiones, el coste por clic medio, el coste por día y la posición probable del anuncio para cada término clave.

\section{Google versus Overture}

El mercado de las empresas suministradoras de enlaces patrocinados está claramente liderado por Overture (Yahoo) y Google, con más de 100.000 clientes cada una, aunque existen también otras compañías importantes de $P P C$, como LookSmart, eSpotting, FindWhat y Kanoodle.

Las principales diferencias entre los dos líderes se pueden encontrar en aspectos como la pre-

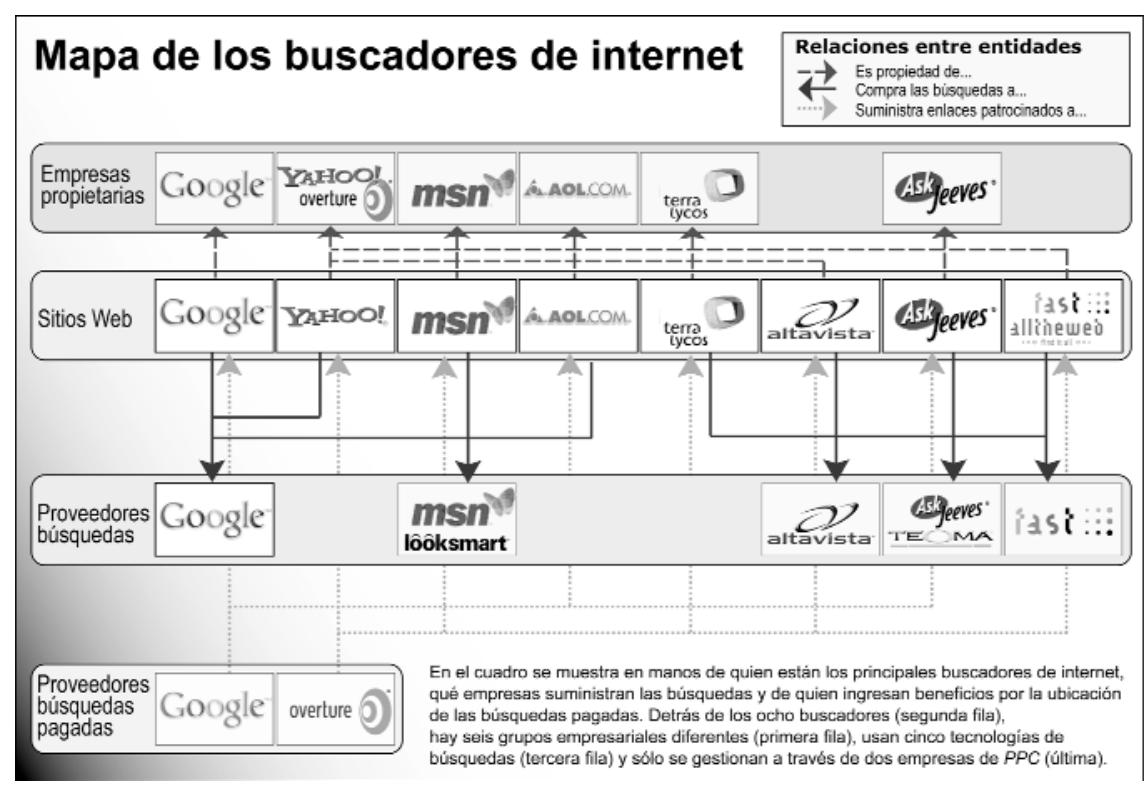

sentación de los resultados, la relevancia que les otorgan y la manera de selección de los términos clave del cliente.

Google separa escrupulosamente los enlaces no pagados de los pagados al situar éstos específicamente en la zona derecha de la página, enmarcados en un fondo de color diferente al resto. Esta estrategia responde a la pretensión de mostrar unos resultados objetivos en los que no interfiera la publicidad.

«En el mercado de las búsquedas ha sido determinante la calidad del servicio ofrecido: se trata de ser el mejor tecnológicamente y disponer los resultados obedeciendo a criterios objetivos»

Otros sitios web de búsquedas han optado por sistemas de visualización menos claros que a menudo pueden crear confusión entre los usuarios. Overture inserta a sus clientes en las listas de resultados del buscador en posiciones preferentes, por encima incluso de los webs que no pagan por publicidad. Se especifica que el resultado es patrocinado con distintas etiquetas que, dependiendo del sitio web, a veces son casi imperceptibles. En definitiva, cuando un usuario busca un término en uno de los buscadores gestionados por la empresa adquirida recientemente por $\mathrm{Ya}$ hoo, primero aparecen varios enlaces pagados por sus clientes y a continuación los que provienen del motor de búsqueda del sitio.

Un segundo elemento diferenciador de Google es que también incorpora criterios que benefician al usuario en la ordenación de la publicidad. El ranking es determinado por la combinación del $C P C$ (coste por clic) y el $C P R$ (porcentaje de clics). Si el anuncio es irrelevante para los usuarios, entonces no harán clic en él. Esto provoca que se mueva a una posición inferior. Los anuncios relevantes, por otra parte, escalarán puestos sin incrementar sus costes.

Un último aspecto diferenciador es la mayor automatización del proceso en Google frente a la mayor intervención humana en Overture. Esta última firma realiza recomendaciones a sus anunciantes sobre los términos por los que pujan respecto a los contenidos, servicios, o productos que desean publicitar, pero lo hace a través de un análisis realizado por profesiona- 

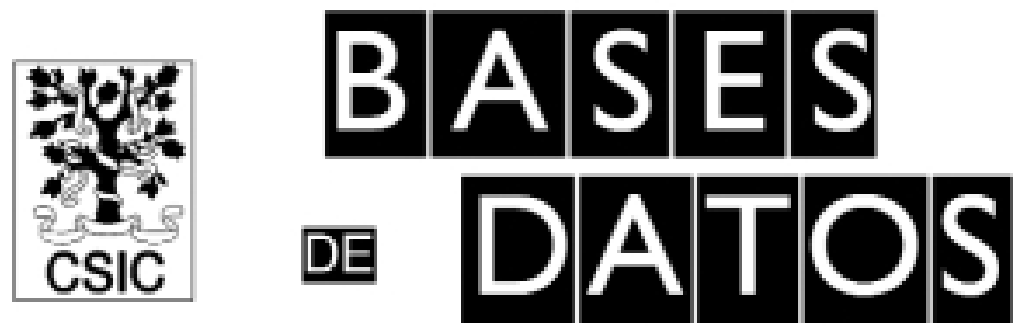

Consejo Superior de Investigaciones Cientificas

- CIREIC: Catálogo colectivo de Librus y Revistas existentes en las bëbliotecas del CSIC. Su temítica es multidisciplinar.

Volumen L.lhros: 1.400,000

Volumen Revistase 74000 Referencias

- ISOC: Base de dalos referencial que recoge y amaliza mís de 1,600 revistas españolas relativas a Humanidades y Ciencias Sociales. Se subdivide, según las distintas áreas temáticas, en:

- ISOC - Amćrica Latina

- ISOC - Antropologia Social

- ISOC - Edacación

- 15OC-Aryucología

- ISOC - Filosofía

- ISOC - Arte

- ISOC - Geogratia, Urbanismo y Aryajitectura

- ISOC - Biblioteror

- ISOC - Historia

- ISOC-Dereche

- ISOC - Lengua y Literatura

- ISOC - Economi

- ISOC - Psicologí

Volumen: 450.000 Referencias

- ISOC - Sociología y CC. Politicas.

Crecimiento Anual: 35 1000 Referencias

- MME: Base de dadus referencial que recoge y unaliza más de 330 revisas médicas espabolas. Volumen: 210.000 Referencias

Crecinaiento Anual: 7,000 Referencias

- ICrT: Base de dafos referencial que recoge y analiza mals de 600 publicaciones periódicas españolas, dentro de los campos de la Ciencia y la Tecnologia.

Volumen: 157.000 Referencias

Crecimiento Anual: 10.000 Referencias

- DATRI (Transferencia de resultados de investigación de la Red OTRLOTT).

Volumen: 8.000 Referencias

Crecinaiento Anual: 1.500 Referencias

- Dates octubre 2003

- EN línea

- CD ROM

CENTRO DE INFORMACIÓN

Y DOCUMENTACIÓN CIENTIFICA (CINDOC)

UNIDAD DE DISTRIBUCIÓN DE BASES DE DATOS

Joaquin Costa, 22

28002 MADRID

Teléfono: 9156354 82 / 87 / 88

Fax: 915642644

Correo electrónico: sdiecindoc.csic.es

Internet: $h t t p=/ / w w w . c i n d o c . c s i c . e s$ 
les. Nick Hynes, presidente europeo de Overture, con motivo de la entrada de la compañía en España el pasado octubre, explicó que un equipo se ocupa de que los contenidos de la web de sus clientes se ajusten a los criterios de búsqueda. "Sólo si pasa este criterio de calidad y relevancia el sitio entra en la lista ofrecida por los buscadores de pago, algo que no se da en Google, que usa el control mediante máquinas y algoritmos, no mediante personas", agregó.

Según Hynes, su empresa paga a los portales, directorios o buscadores el $63 \%$ de sus ganancias. Los anunciantes logran un mejor posicionamiento en las páginas de resultados de búsquedas, donde el mismo usuario ha hecho la segmentación; los portales no deben preocuparse de la estructura comercial ni de la tecnología necesaria para mantener una base de datos separada de enlaces comerciales y además cobran por cada búsqueda que realizan los usuarios mientras que Overture se lleva su parte como mayorista de publicidad.

\section{Algunas previsiones}

La situación del sector de los buscadores de internet en 2003 es previsible que se prolongue en los primeros meses de 2004 a la espera de algunos sucesos que los analistas dan por seguros: la salida a bolsa de Google; la no renovación por parte de Yahoo del contrato de suministro de búsquedas con Google; y el lanzamiento del nuevo motor de Microsoft.

Las especulaciones sobre qué pasará a partir de la cotización en el mercado bursátil del buscador número uno son de todos los colores. Las previsiones más optimistas la sitúan en un nuevo ciclo de euforia tecnológica que la actual recuperación de las empresas con negocios en la Red parece alentar. Las más pesimistas recurren al recuerdo de Netscape: Google tam-

\section{IweTel}

Es un foro electrónico de debate, puesto en marcha por EPI - El profesional de la información, sobre información, documentación, biblioteconomía y sus tecnologías.

En la actualidad cuenta con más de 4.800 suscriptores.

Para suscribirse a IweTel hay que enviar a la dirección:

listserv@ listserv.rediris.es

un mensaje en cuyo cuerpo figure:

subscribe iwetel Nombre Apellido

Se puede participar en IweTel remitiendo los mensajes a:

iwetel@ listserv.rediris.es

Más información en:

http://www.rediris.es/list/info/iwetel.html

bién es una compañía inicialmente pequeña que ha desarrollado una tecnología de gran éxito y que se enfrenta al gigante Microsoft, con todas sus consecuencias. Como es bien sabido, la compañía de Bill Gates, gracias a las ventajas de disponer del sistema operativo dominante, consiguió barrer del mercado al en otros tiempos popular navegador.

\section{"Las consecuencias del éxito o no de la salida a bolsa de Go- ogle, las réplicas de Yahoo y, en especial, el nuevo buscador de Microsoft, pueden marcar el futuro in- mediato de los bus- cadores de la Red»}

En el sector de las búsquedas hasta ahora ha sido determinante la calidad del servicio ofrecido: se trata de ser el mejor tecnológicamente. Si aparece un buscador mejor que el actual líder, nada podrá evitar que arrebate a éste su posición hegemónica, siempre que la presión que pueden ejercer algunas marcas no distorsione el mercado y provoque otros casos Netscape.

La tecnología de los motores de búsqueda, en opinión de los ex- pertos, resulta aún demasiado imperfecta, por lo que caben esperar todavía avances significativos. Internet ha sido hasta ahora un mercado muy dinámico e incierto, con apariciones constantes de nuevas opciones, marcas y servicios que tanto han despuntado en la Red como han desaparecido, por lo que no tiene porqué no seguir sucediendo. Un ejemplo de una nueva tendencia, todavía minoritaria para el gran público pero con gran potencial de crecimiento es la que constituye la nueva generación de buscadores que organizan visualmente la información, como Grokker o Kartoo. El tiempo dirá si opciones innovadoras de este tipo conseguirán algún trozo importante del pastel que se reparten hoy casi en exclusiva los tres grandes.

En cualquier caso, las consecuencias del éxito o no de la salida a bolsa de Google, y las réplicas de Yahoo y en especial la muy esperada de Microsoft, pueden marcar el futuro inmediato de los buscadores de la Red. Claro que, tratándose de internet, las sorpresas siempre forman parte del guión.

Jordi Grau Moracho y Javier Gua-
llar Delgado
jordi@grau.com
jguallard@uoc.edu

